\title{
Presentations of major peripheral arterial disease and risk of major outcomes in patients with type 2 diabetes: results from the ADVANCE-ON study
}

Kamel Mohammedi ${ }^{1^{*}}$, Mark Woodward ${ }^{1,2,3}$, Yoichiro Hirakawa ${ }^{1}$, Sophia Zoungas ${ }^{1,4}$, Stephen Colagiuri ${ }^{5}$, Pavel Hamet ${ }^{6}$, Stephen Harrap ${ }^{7}$, Neil Poulter ${ }^{8}$, David R. Matthews ${ }^{9}$, Michel Marre ${ }^{10,11,12}$, John Chalmers ${ }^{1}$ and on behalf of the ADVANCE Collaborative Group

\begin{abstract}
Background: Peripheral arterial disease (PAD) is known to be associated with high cardiovascular risk, but the individual impact of PAD presentations on risk of macrovascular and microvascular events has not been reliably compared in patients with type 2 diabetes. We aimed to evaluate the impact of major PAD, and its different presentations, on the 10-year risk of death, major macrovascular events, and major clinical microvascular events in these patients.
\end{abstract}

Methods: Participants in the action in diabetes and vascular disease: PreterAx and DiamicroN modified-release controlled evaluation (ADVANCE) trial and the ADVANCE-ON post-trial study were followed for a median of 5.0 (in-trial), 5.4 (post-trial), and 9.9 (overall) years. Major PAD at baseline was subdivided into lower-extremity chronic ulceration or amputation secondary to vascular disease and history of peripheral revascularization by angioplasty or surgery.

Results: Among 11,140 participants, 516 (4.6\%) had major PAD at baseline: 300 (2.7 \%) had lower-extremity ulceration or amputation alone, 190 (1.7\%) had peripheral revascularization alone, and 26 (0.2\%) had both presentations. All-cause mortality, major macrovascular events, and major clinical microvascular events occurred in 2265 (20.3\%), 2166 (19.4\%), and 807 (7.2\%) participants, respectively. Compared to those without PAD, patients with major PAD had increased rates of all-cause mortality ( $H R 1.35,95 \% \mathrm{Cl} 1.15-1.60, p=0.0004)$, and major macrovascular events (1.47 [1.23-1.75], $\mathrm{p}<0.0001$ ), after multiple adjustments for region of origin, cardiovascular risk factors and treatments, peripheral neuropathy markers, and randomized treatments. We have also observed a trend toward an association of baseline PAD with risk of major clinical microvascular events [1.31 (0.96-1.78), $p=0.09]$. These associations were comparable for patients with a lower-extremity ulceration or amputation and for those with a history of peripheral revascularization. Furthermore, the risk of retinal photocoagulation or blindness, but not renal events, increased in patients with lower-extremity ulceration or amputation [1.53 (1.01-2.30), $p=0.04]$.

Conclusions: Lower-extremity ulceration or amputation, and peripheral revascularization both increased the risks of death and cardiovascular events, but only lower-extremity ulceration or amputation increased the risk of severe retinopathy in patients with type 2 diabetes. Screening for major PAD and its management remain crucial for cardiovascular prevention in patients with type 2 diabetes (ClinicalTrials.gov number, NCT00949286).

Keywords: Type 2 diabetes, Peripheral arterial disease, Lower-extremity ulceration, Lower-extremity amputation, Mortality, Cardiovascular diseases, Major macrovascular events, Diabetic retinopathy

\footnotetext{
*Correspondence: kmohammedi@georgeinstitute.org.au

${ }^{1}$ The George Institute for Global Health, University of Sydney, PO Box

M201, Missenden road, Camperdown, Sydney, NSW 2050, Australia

Full list of author information is available at the end of the article
} 


\section{Background}

Peripheral arterial disease (PAD) is a public health problem across the world with a significant impact on healthcare and a high economic burden [1-5]. It is associated with an increased risk of cardiovascular disease [6-8], and is particularly common in patients with type 2 diabetes [9-12]. Previous studies have shown poor survival and cardiovascular outcomes in patients with both type 2 diabetes and PAD [13-15]. However, the individual impact of different presentations of major PAD on survival and risk of major macrovascular and microvascular events in patients with type 2 diabetes has not been reliably evaluated in long-term prospective studies. The Action in Diabetes and Vascular Disease: PreterAx and DiamicroN Modified-Release Controlled Evaluation (ADVANCE) trial followed patients with type 2 diabetes for a median of 5.0 years, and the ADVANCE-ON posttrial observational study followed them up for further 5.4 years, to a median overall period of 9.9 years $[16,17]$. The current investigation aimed to examine the impact of major PAD at baseline on the risk of mortality and major macrovascular and microvascular outcomes in patients with type 2 diabetes across the full 9.9 years, and to compare the effects on these outcomes, of presentation with lower-extremity ulceration or amputation on one hand, as against presentation with previous peripheral revascularization on the other.

\section{Methods}

\section{Population study}

ADVANCE was an international randomized trial in 11,140 patients with type 2 diabetes with the objectives to test the effect of intensive glucose control and blood pressure treatment on the incidence of major microvascular and macrovacular events [16]. Participants with type 2 diabetes at high risk of cardiovascular events were randomly assigned to a gliclazide (modified release)-based intensive glucose-control regimen, aiming to achieve an HbA1c $\leq 6.5 \%$, or to standard glucose control, with targets and regimens based on local guidelines. Participants were also randomly assigned to a fixed-dose combination of perindopril $(4 \mathrm{mg})$ and indapamide $(1.25 \mathrm{mg})$ or matching placebo. Subsequently 8494 surviving participants were enrolled in the post-trial observational evaluation, ADVANCE-ON, study. Design, characteristics of participants, and the main results of both studies have been previously published [16-19].

\section{Definition of peripheral arterial disease}

Major PAD at baseline was defined, as in the ADVANCE study, as lower-extremity chronic ulceration (at least 6 weeks) or amputation below the knee (of at least one toe), secondary to vascular disease, or history of peripheral revascularization procedure by angioplasty or surgery [16]. Two presentations of major PAD were compared: lower-extremity ulceration or amputation versus peripheral revascularization. Patients with both presentations $(n=26)$ were excluded in the comparison of the outcomes according to each PAD presentation.

\section{Definition of outcomes}

There were three primary outcomes: all-cause mortality, major macrovascular events (a composite of nonfatal myocardial infarction, nonfatal stroke, or cardiovascular death), and major clinical microvascular events (a composite of end-stage renal disease (ESRD), defined as requirement for renal-replacement therapy; death induced by renal disease; requirement for retinal photocoagulation; or diabetes-related blindness in either eye). The secondary outcomes were cardiovascular death, fatal or nonfatal myocardial infarction, fatal or nonfatal stroke, ESRD or renal death, and requirement for retinal photocoagulation or blindness. Outcomes were adjudicated by an independent End Point Adjudication Committee in the ADVANCE trial, through to the end of randomized treatment, and were reported by investigators without adjudication in the ADVANCE-ON study, in accord with its pre-specified protocol [17]. Information about the occurrence of study outcomes and of all serious adverse events was reported at the time of occurrence between visits. When study outcomes or serious adverse events occured, the responsible investigator of each centre ensured that the event was reported immediately by completing a serious adverse events form. The data and safety monitoring committee regularly reviewed all such events for each centre.

\section{Statistical analyses}

Categorical variables were presented as the number of patients with the corresponding percentage. Continuous variables were expressed as mean (SD) or median (interquartile range) for those with skewed distribution. Characteristics of participants according to major PAD status (absence vs. presence) and presentation (lower-extremity ulceration or amputation vs. peripheral revascularization) were compared at baseline using Chi squared, ANOVA, or Wilcoxon tests. Cumulative incidence curves were used to plot survival (outcome-free) rates during follow-up. Survival curves were compared using the logrank test. Cox proportional hazards survival regression models were fitted to examine the effect of baseline history of major PAD and its presentations on time-related survival (outcome-free) rates. Hazard ratios (HR), with associated $95 \%$ confidence intervals $(\mathrm{CI})$, were estimated according to major PAD status at baseline. Basic (model 1: region of origin, sex, age, body mass index, systolic 
blood pressure, history of ever smoking and study allocations), and full (model 2: model 1 plus duration of diabetes, HbA1c, waist circumference, heart rate, diastolic blood pressure, disturbance of 10 -g monofilament sensation, absence of ankle and knee reflexes, estimated glomerular filtration rate (eGFR) [and its square for macrovascular analyses], total-, and HDL-cholesterol, triglycerides, use of antihypertensive, lipid lowering and antiplatelet drugs, and history of current alcohol drinking) adjustments were performed. Participants with a history of macrovascular disease (defined as the presence at baseline, of myocardial infarction, stroke, coronary artery bypass graft, percutaneous transluminal coronary angioplasty, hospital admission for unstable angina or transient ischaemic attack) were excluded in sensitivity analyses. $\mathrm{p}<0.05$ was considered as significant. Statistical analyses were performed using SAS software, version 9.3 (SAS Institute, http://www.sas.com).

\section{Results}

\section{Clinical characteristics at baseline}

Among 11,140 patients randomized at baseline, 516 (4.6\%) had a history of major PAD. Clinical characteristics of participants at baseline are shown in Additional file 1: Table S1. Participants with major PAD at baseline, compared to those without PAD, were older, and more frequently men and from established market economies. They had higher body mass index, waist circumference, and urinary albumin-creatinine ratio, and lower diastolic blood pressure, eGFR, and serum total and HDL-cholesterol. They were also more likely to use lipid lowering and antiplatelet drugs, and to have a history of ever smoking or current alcohol drinking. Patients with major PAD had also more frequent disturbance of $10-\mathrm{g}$ monofilament sensation and absence of ankle and knee reflexes.

\section{Incidence of outcomes during follow-up according to the history of major PAD at baseline}

The median (interquartile interval) duration of overall follow-up was 9.9 (5.6-10.9) years. All-cause mortality, major macrovascular events, cardiovascular death, and major clinical microvascular events occurred in 2265 (20.3\%), 2166 (19.4\%), 988 (8.9\%), and 807 (7.2\%) participants, respectively. The cumulative incidence of all-cause mortality, major macrovascular events, cardiovascular death, and fatal or non-fatal myocardial infarction were higher in participants with history of major PAD at baseline compared to those without PAD ( $\mathrm{p}<0.0001$ for all): Fig. 1; Table 1. Significant associations of PAD with the risk of all-cause mortality (HR 1.35, $95 \%$ CI 1.15-1.60, $\mathrm{p}=0.0004)$, major macrovascular events (HR 1.47, 95 \% CI 1.23-1.75, p < 0.0001), cardiovascular death (HR 1.75, 95 \% CI 1.39-2.21, p < 0.0001), and myocardial infarction (HR 1.58, 95 \% CI 1.19-2.09, $\mathrm{p}=0.001$ ) persisted after adjustment for all potential confounding variables. A trend toward an association of major PAD with requirement of retinal photocoagulation or blindness persisted in the fully adjusted model (HR 1.39, $95 \%$ CI 0.99-1.95, $\mathrm{p}=0.05$ ). However, we did not observe association of major PAD at baseline with the risk of ESRD or renal death (Table 1).

\section{Incidence of outcomes during follow-up according to different presentations of major PAD at baseline} Lower-extremity ulceration or amputation, and history of peripheral revascularization, not concurrently occurring, were established at baseline in 300 (2.7\%) and 190 $(1.7 \%)$ participants, respectively $(\mathrm{p}<0.0001)$. Only 26 $(0.2 \%)$ patients had both presentations of major PAD at baseline. Compared to those with lower-extremity ulceration or amputation, participants with history of peripheral revascularization, were more frequently men and from established market economies (Table 2). They had a shorter duration of diabetes, lower eGFR and serum total cholesterol, were more likely to use lipid lowering and antiplatelet drugs, and to have a history of ever smoking or current alcohol drinking. The cumulative incidence of all-cause mortality, major macrovascular events, cardiovascular death, and fatal and non-fatal myocardial infarction increased comparably in subjects with different presentations of major PAD, compared to those without PAD (Fig. 2; Table 3). Associations of both presentations with all-cause mortality, major macrovascular events, cardiovascular death, and myocardial infarction were confirmed in the fully adjusted model 2 . Furthermore, lower-extremity ulceration or amputation was significantly associated with a higher risk of blindness or of requirement for retinal photocoagulation (HR 1.53, $95 \%$ CI $1.01-2.30, \mathrm{p}=0.04)$. A non-significant trend toward an association of lower-extremity ulceration or amputation was also observed with the risk of major clinical microvascular events (HR 1.44, 95 \% CI 0.98-2.11, $\mathrm{p}=0.06$ ). However, no association of lower-extremity ulceration or amputation was observed with the risk of ESRD or renal death. The risks for outcomes according to each presentation of major PAD were comparable for mortality and major macrovascular outcomes, but not for retinal complications.

\section{Sensitivity analyses}

Major PAD was established in 283 (3.7\%) patients among 7679 participants free from history of macrovascular disease at baseline. In this subset of participants, the association of major PAD with the risk of all-cause mortality, major macrovascular events and cardiovascular death remained significant (Additional file 1: Table S2). 


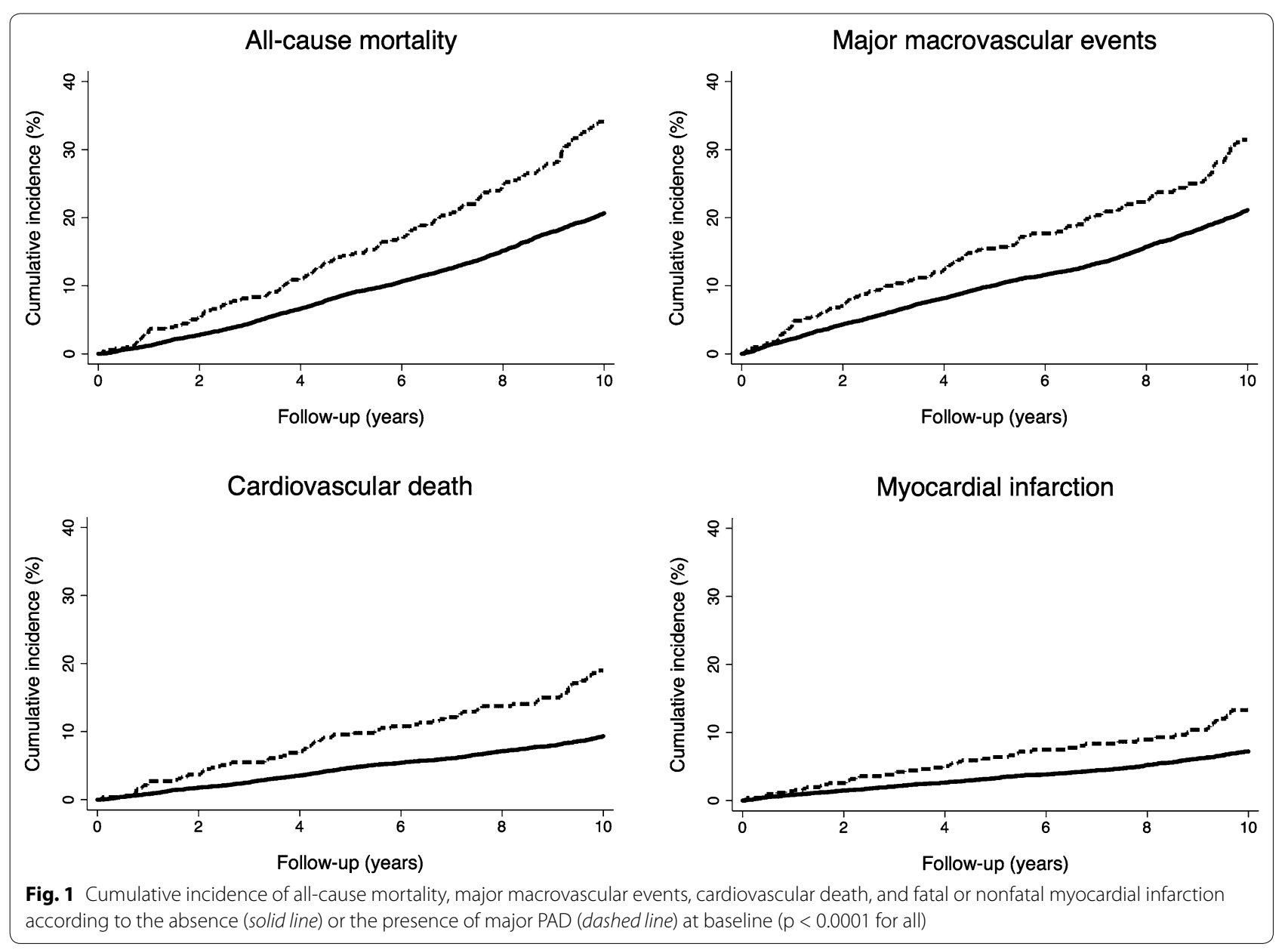

Table 1 Hazard ratios for outcomes during follow-up by history of major PAD at baseline

\begin{tabular}{|c|c|c|c|c|c|c|c|c|}
\hline & \multicolumn{2}{|c|}{ Baseline history of major PAD } & \multicolumn{6}{|c|}{ Hazard ratios (major PAD vs. not) } \\
\hline & \multirow[t]{2}{*}{ No $(n=10,624)$} & \multirow[t]{2}{*}{ Yes $(n=516)$} & \multicolumn{3}{|c|}{ Model 1} & \multicolumn{3}{|c|}{ Model 2} \\
\hline & & & HR & $95 \% \mathrm{Cl}$ & $\mathrm{p}$ & HR & $95 \% \mathrm{Cl}$ & $\mathbf{p}$ \\
\hline All-cause mortality, n (\%) & $2101(19.8)$ & $164(31.8)$ & 1.57 & $1.33-1.84$ & $<0.0001$ & 1.35 & $1.15-1.60$ & 0.0004 \\
\hline Major macrovascular events, n (\%) & $2025(19.1)$ & $141(27.3)$ & 1.59 & $1.34-1.88$ & $<0.0001$ & 1.47 & $1.23-1.75$ & $<0.0001$ \\
\hline Cardiovascular death, n (\%) & $904(8.5)$ & $84(16.3)$ & 1.99 & $1.59-2.49$ & $<0.0001$ & 1.75 & $1.39-2.21$ & $<0.0001$ \\
\hline Myocardial infarction, n (\%) & $665(6.3)$ & $57(11.0)$ & 1.76 & $1.34-2.31$ & $<0.0001$ & 1.58 & $1.19-2.09$ & 0.001 \\
\hline Stroke, n (\%) & $924(8.7)$ & $44(8.5)$ & 1.24 & $0.92-1.68$ & 0.16 & 1.19 & $0.87-1.62$ & 0.28 \\
\hline $\begin{array}{l}\text { Major clinical microvascular events, } \\
\text { n (\%) }\end{array}$ & $763(7.2)$ & $44(8.5)$ & 1.43 & $1.05-1.94$ & 0.02 & 1.31 & $0.96-1.78$ & 0.09 \\
\hline $\begin{array}{l}\text { Retinal photocoagulation or blindness, } \\
\mathrm{n}(\%)\end{array}$ & $632(5.9)$ & $37(7.2)$ & 1.48 & $1.06-2.07$ & 0.02 & 1.39 & $0.99-1.95$ & 0.05 \\
\hline $\begin{array}{l}\text { End-stage renal disease or renal death, } \\
\text { n (\%) }\end{array}$ & $160(1.5)$ & $8(1.6)$ & 1.15 & $0.56-2.34$ & 0.71 & 0.96 & $0.46-1.97$ & 0.90 \\
\hline
\end{tabular}

Model 1: adjusted for region of origin, sex, age, body mass index, systolic blood pressure, history of ever smoking, and study allocations. Model 2: adjusted as in model 1 plus duration of diabetes, $\mathrm{HbA1c}$, waist circumference, heart rate, diastolic blood pressure, disturbance of 10-g monofilament sensation, absence of ankle and knee reflexes, estimated glomerular filtration rate (and its square for macrovascular analyses), total-, and HDL-cholesterol, triglycerides, use of antihypertensive, lipid lowering and antiplatelet drugs, and history of current alcohol drinking. $\mathrm{p}<0.05$ was significant 
Table 2 Clinical characteristics of participants according to different presentations of major PAD at baseline

\begin{tabular}{|c|c|c|c|}
\hline & $\begin{array}{l}\text { Lower-extremity ulceration } \\
\text { or amputation }(\mathrm{n}=300)\end{array}$ & $\begin{array}{l}\text { Peripheral revascularization } \\
(\mathrm{n}=190)\end{array}$ & $\mathrm{p}$ \\
\hline Male sex, n (\%) & $177(59.0)$ & $140(73.7)$ & 0.0009 \\
\hline Region of origin: Asia, n (\%) & $81(27.0)$ & $12(6.3)$ & $<0.0001$ \\
\hline Region of origin: established market economies, n (\%) & $158(52.7)$ & $135(71.0)$ & \\
\hline Region of origin: Eastern Europe, n (\%) & $61(20.3)$ & $43(22.6)$ & \\
\hline Age (years): mean (SD) & $66.2(6.7)$ & $66.6(6.9)$ & 0.55 \\
\hline Duration of diabetes (years): median (Q1, Q3) & $8.0(4.0,12.0)$ & $6.0(3.0,11.0)$ & 0.007 \\
\hline Waist circumference (cm): mean (SD) & $101(14)$ & $102(14)$ & 0.72 \\
\hline Body mass index $\left(\mathrm{kg} / \mathrm{m}^{2}\right)$ : mean (SD) & $29.0(5.9)$ & $28.5(4.4)$ & 0.32 \\
\hline Heart rate (bpm): mean (SD) & $74.5(11.1)$ & $72.4(13.2)$ & 0.06 \\
\hline Systolic blood pressure (mmHg): mean (SD) & $145(22)$ & $144(22)$ & 0.76 \\
\hline Diastolic blood pressure (mmHg): mean (SD) & $80(11)$ & $78(10)$ & 0.13 \\
\hline Use of antihypertensive treatment, n (\%) & $210(70.0)$ & $143(75.3)$ & 0.21 \\
\hline Disturbance of 10-g monofilament sensation, n (\%) & $59(19.7)$ & $30(15.8)$ & 0.28 \\
\hline Absence of ankle reflex, n (\%) & $102(34.0)$ & $51(26.8)$ & 0.10 \\
\hline Absence of knee reflex, n (\%) & $54(18.0)$ & $19(10.0)$ & 0.02 \\
\hline HbA1c (\%): median (Q1, Q3) & $7.3(6.5,8.7)$ & $7.0(6.4,7.9)$ & 0.18 \\
\hline HbA1c (mmol/mol): median (Q1, Q3) & $56(47,72)$ & $53(46,63)$ & \\
\hline $\mathrm{eGFR}\left(\mathrm{ml} / \mathrm{min} / 1.73 \mathrm{~m}^{2}\right)$ & $74(18)$ & $70(18)$ & 0.01 \\
\hline $\begin{array}{l}\text { Urinary albumin to creatinine ratio }(\mu \mathrm{g} / \mathrm{mg}) \text { : } \\
\text { median }(\mathrm{Q} 1, \mathrm{Q} 3)\end{array}$ & $19(9,64)$ & $14(6,55)$ & 0.06 \\
\hline Serum total cholesterol (mmol/l): mean (SD) & $5.2(1.1)$ & $4.8(1.0)$ & 0.0004 \\
\hline Serum HDL cholesterol (mmol/l): mean (SD) & $1.2(0.3)$ & $1.2(0.3)$ & 0.37 \\
\hline Serum triglycerides (mmol/l): median (Q1, Q3) & $1.7(1.2,2.5)$ & $1.8(1.2,2.3)$ & 0.56 \\
\hline Use of lipid lowering drugs, n (\%) & $103(34.3)$ & $126(66.3)$ & $<0.0001$ \\
\hline Use of antiplatelet drugs, n (\%) & $139(46.3)$ & $143(75.3)$ & $<0.0001$ \\
\hline History of current smoking, n (\%) & $47(15.7)$ & $30(15.8)$ & 0.97 \\
\hline History of ever smoking, n (\%) & $153(51.0)$ & $133(70.0)$ & $<0.0001$ \\
\hline History of current drinking, n (\%) & $86(28.7)$ & $89(46.8)$ & $<0.0001$ \\
\hline
\end{tabular}

Comparison of qualitative and quantitative parameters were performed using Chi square and ANOVA tests, respectively. Wilcoxon test was used for variables with skewed distribution (duration of diabetes, $\mathrm{HbA} 1 \mathrm{c}$, urinary albumin-creatinine ratio and triglycerides). $\mathrm{p}<0.05$ was significant

Asia: Philippines, China, Malaysia, India; Established market economies: Australia, Canada, France, Germany, Ireland, Italy, Netherlands, New Zealand, United Kingdom; Eastern Europe: the Czech Republic, Estonia, Hungary, Lithuania, Poland, Russia, Slovakia. eGFR, estimated Glomerular Filtration Rate computed by the Chronic Kidney Disease Epidemiology Collaboration equation

History of current drinking was defined as consumption of alcohol at least once a week for most weeks of the previous year

Lower-extremity ulceration or amputation, and the history of peripheral revascularization also remained significantly associated with increased risk for all-cause mortality (HR 1.60, $95 \%$ CI 1.22-2.10, $\mathrm{p}=0.0007$, and HR 1.57, $95 \%$ CI 1.06-2.34, $\mathrm{p}=0.02$, respectively) and major macrovascular events (HR 1.63, $95 \%$ CI 1.20-2.21, $\mathrm{p}=0.002$, and HR 1.80, $95 \%$ CI 1.14-2.86, $\mathrm{p}=0.01$, respectively).

\section{Discussion}

We have investigated the risk of vascular outcomes with major PAD, and its different presentations, in patients with type 2 diabetes followed for 10 years in the ADVANCE and ADVANCE-ON studies. Major PAD at baseline was associated with increased risk of all-cause mortality, major macrovascular events, cardiovascular death, and myocardial infarction. Lower-extremity ulceration or amputation, and the history of peripheral revascularization displayed similar risks of death and major cardiovascular events. However, lower-extremity ulceration or amputation, but not peripheral revascularization, was also associated with increased risk of retinal photocoagulation or blindness.

\section{History of PAD and risk of macrovascular events}

In line with our findings, poor survival and macrovascular outcomes associated with PAD were reported in other prospective studies of patients with type 2 diabetes, even 

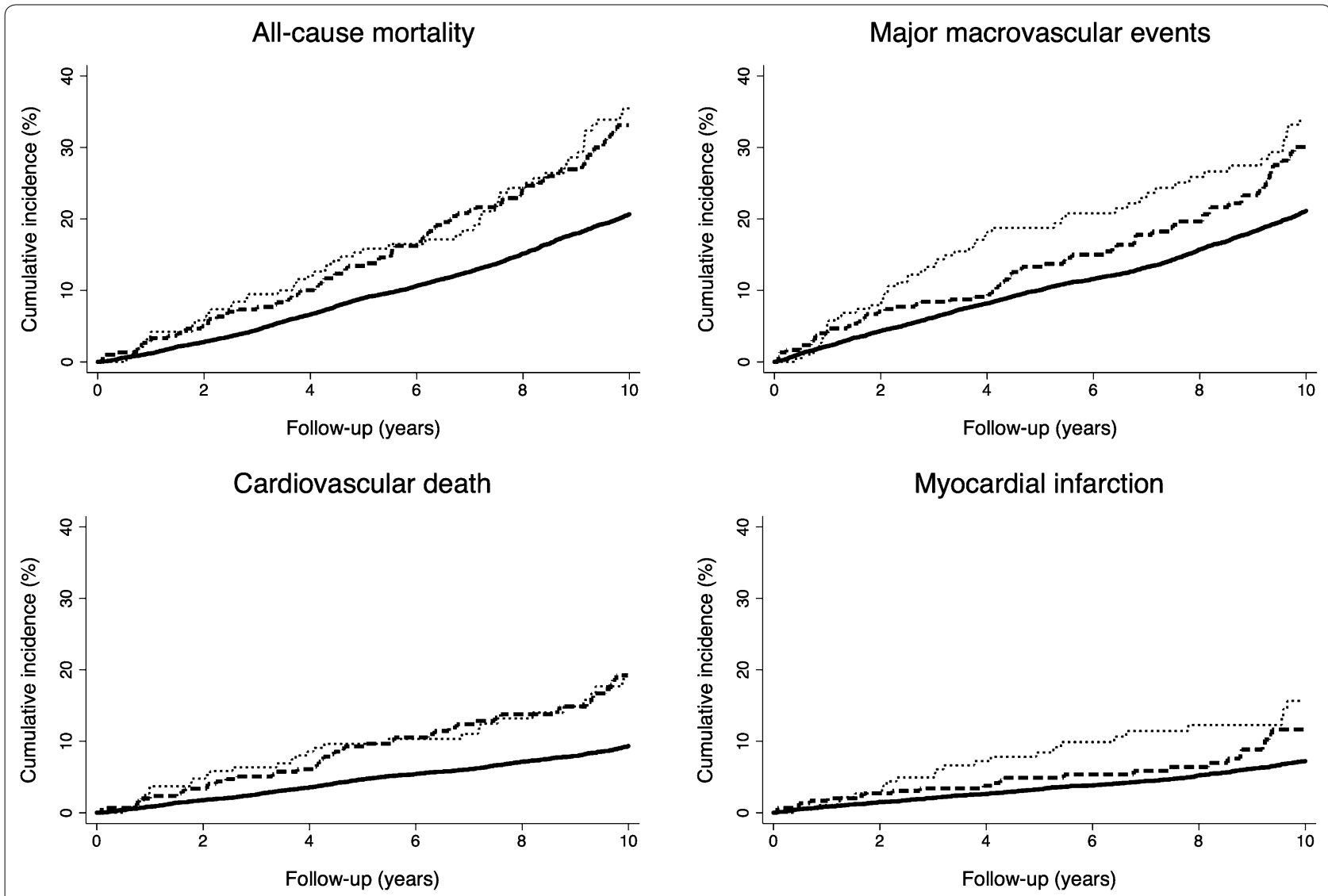

Fig. 2 Cumulative incidence of all-cause mortality, major macrovascular events, cardiovascular death, and fatal or nonfatal myocardial infarction according to the absence (solid line) or the presence of lower-extremity ulceration or amputation (dashed line) and peripheral revascularization (dotted line) at baseline ( $p<0.0001$ for all)

in those who have not been treated with hypoglycaemic drugs [13-15, 20]. In the PROspective pioglitAzone clinical trial in macrovascular events (PROactive) trial, baseline PAD was associated with high risk of all-cause mortality, and macrovascular complications in 5238 patients with type 2 diabetes during a mean follow-up of 2.9 years [14]. In the Bypass Angioplasty Revascularization Investigation 2 Diabetes (BARI 2D) trial, low anklebrachial index or non-compressible artery were associated with increased risks of death and major cardiovascular events in 2368 patients with type 2 diabetes followed for an average of 4.3 years [15]. Our larger study demonstrates that the association of major PAD with death and major macrovascular events persisted during 10 years of follow-up, even after adjustment for major risk factors. Furthermore, these associations persisted in patients free for any history of macrovascular disease at baseline.

\section{Presentations of PAD and risk of macrovascular events}

As distal PAD is known to be associated with a high rate of ulceration and amputation, and is rarely accessible for revascularization $[9,21]$, it is tempting to speculate that our patients with history of lower-extremity ulceration or amputation, were more likely to have a distal than a proximal origin for their PAD. The determination of PAD localizations differs among studies, but angiography and Doppler ultrasound are still considered as the key imaging methods [21, 22]. A recent study has reported a semi-quantitative ultrasonographic score to evaluate the association of PAD severity and localizations with cardiovascular risk factors and adverse events [23]. This showed that the magnitude of the association of PAD with diabetes was greater with distal than proximal localizations. Major PAD presentations had different clinical characteristics in our study. Lower-extremity ulceration or amputation was more common than peripheral revascularization, and associated with a longer duration of diabetes. Furthermore, lower-extremity ulceration, amputation, and peripheral revascularization occurred concurrently in only 26 patients, suggesting that these presentations of major PAD have different origins. Comparison of survival, macrovascular and microvascular 
Table 3 Hazard ratios for outcomes during follow-up according to different presentations of major PAD at baseline

\begin{tabular}{|c|c|c|c|c|c|c|c|}
\hline & \multirow[t]{2}{*}{$\begin{array}{l}\text { Absence } \\
\text { of PAD }\end{array}$} & \multirow[t]{2}{*}{$\begin{array}{l}\text { Lower-extremity } \\
\text { ulceration or } \\
\text { amputation }\end{array}$} & \multirow[t]{2}{*}{$\begin{array}{l}\text { Peripheral } \\
\text { revascularization }\end{array}$} & \multicolumn{2}{|c|}{$\begin{array}{l}\text { Lower-extremity ulceration } \\
\text { or amputation } \\
\text { (vs. absent PAD) }\end{array}$} & \multicolumn{2}{|c|}{$\begin{array}{l}\text { Peripheral revasculari- } \\
\text { zation } \\
\text { (vs. absent PAD) }\end{array}$} \\
\hline & & & & $\mathrm{HR}(95 \% \mathrm{Cl})$ & p & $\mathrm{HR}(95 \% \mathrm{Cl})$ & $p$ \\
\hline All-cause mortality, n (\%) & $2101(19.8)$ & $92(30.7)$ & $63(33.2)$ & $1.39(1.12-1.72)$ & 0.003 & $1.31(1.01-1.70)$ & 0.04 \\
\hline $\begin{array}{l}\text { Major macrovascular events, } \\
\text { n (\%) }\end{array}$ & $2025(19.1)$ & $78(26.0)$ & $56(29.5)$ & $1.39(1.11-1.75)$ & 0.005 & $1.64(1.25-2.16)$ & 0.0004 \\
\hline Cardiovascular death, n (\%) & $904(8.5)$ & $50(16.7)$ & $29(15.3)$ & $1.91(1.43-2.54)$ & $<0.0001$ & $1.52(1.04-2.23)$ & 0.03 \\
\hline Myocardial infarction, n (\%) & $665(6.3)$ & $29(9.7)$ & $25(13.2)$ & $1.50(1.03-2.19)$ & 0.03 & $1.73(1.14-2.62)$ & 0.01 \\
\hline Stroke, n (\%) & $924(8.7)$ & $24(8.0)$ & $18(9.5)$ & $1.02(0.67-1.55)$ & 0.92 & $1.54(0.96-2.48)$ & 0.07 \\
\hline $\begin{array}{l}\text { Major clinical } \\
\text { microvascular events, n (\%) }\end{array}$ & $763(7.2)$ & $28(9.3)$ & $11(5.8)$ & $1.44(0.98-2.11)$ & 0.06 & $0.88(0.48-1.61)$ & 0.68 \\
\hline $\begin{array}{l}\text { Retinal photocoagulation or } \\
\text { blindness, } \mathrm{n}(\%)\end{array}$ & $632(5.9)$ & $24(8.0)$ & $9(4.7)$ & $1.53(1.01-2.30)$ & 0.04 & $0.94(0.48-1.83)$ & 0.85 \\
\hline $\begin{array}{l}\text { End-stage renal disease or } \\
\text { renal death, } \mathrm{n}(\%)\end{array}$ & $160(1.5)$ & $4(1.3)$ & $3(1.6)$ & $0.84(0.31-2.30)$ & 0.74 & $0.94(0.29-3.02)$ & 0.92 \\
\hline
\end{tabular}

Adjusted as in model 2: region of origin, sex, age, duration of diabetes, body mass index, waist circumference, heart rate, systolic and diastolic blood pressure, disturbance of 10-g monofilament sensation, absence of ankle and knee reflexes, $\mathrm{HbA1C}$, estimated glomerular filtration rate (and its square for macrovascular analyses), total-, and HDL-cholesterol, triglycerides, history of ever smoking and current alcohol drinking, use of antihypertensive, lipid lowering and antiplatelet drugs, and study allocations. $p<0.05$ was significant

outcomes according to different presentations of major PAD have not been extensively performed in people with diabetes. Despite decrease in its prevalence and incidence during last decades in some regions in the world $[24,25]$, lower-extremity amputation will still exert an important health burden. Lower-extremity amputation and ulceration were associated with increased risk of major macrovascular events, and of cardiovascular and non-cardiovascular death in patients with diabetes [2629]. The risk of major cardiovascular events was also high in patients with PAD undergoing peripheral revascularization in a general population cohort including $39 \%$ of participants with diabetes [30]. The pathophysiological links by which PAD might predispose to poor vascular outcomes have not yet been fully elucidated in patients with diabetes. The increased formation of advanced glycation end-products (AGE) and its receptors (RAGE) may be a potential pathway linking PAD and other major vascular outcomes. A recent study has shown association of high plasma levels of AGE-RAGE components with increased risk for amputation, PAD, or death in patients with type 2 diabetes [31].

\section{Presentations of PAD and risk of microvascular events}

Importantly, our findings highlight for the first time, the association of lower-extremity ulceration or amputation with the risk of severe eye complications during 10-years follow-up. This is comparable to a recent cross-sectional study, which showed a higher prevalence of PAD in patients with proliferative diabetic retinopathy rather than those with non-proliferative retinopathy [32]. We have also reported recently, association of history of diabetic retinopathy with requirement for laser photocoagulation at baseline with excess risk of major PAD during follow-up in ADVANCE study [33]. Our present findings are unlikely to be explained by a possible effect of peripheral diabetic neuropathy, since associations we have observed persisted despite adjustment for disturbance of 10-g monofilament sensation and absence of ankle and knee reflexes. Furthermore, the distribution of the key neuropathic markers was comparable between the different presentations of major PAD. However, we did not observe an association of either presentation of major PAD with the risk of major renal outcomes. Remarkably, only 3 patients with major PAD at baseline developed ESRD during 10 years of follow-up. Based on their high rate of mortality, patients with both PAD and chronic kidney disease (CKD) may have died before experiencing more advanced renal outcomes [34, 35]. A recent study showed that the 4-year mortality rate of patients with PAD and CKD stages 4 , and 5 was 72 and $78 \%$, respectively [36].

\section{Strengths and limitations}

As compared to previous studies in people with type 2 diabetes, we have observed a lower prevalence of PAD, which may partially be explained by the differences in PAD definitions used $[13,14]$. The main limitation of our investigation is that we have studied patients with symptomatic and severe PAD, and may have underestimated outcomes associated with asymptomatic PAD. However, our work has several strengths including the investigation 
of a large contemporary cohort of patients with type 2 diabetes at high cardiovascular risk, with a comprehensive clinical history of major PAD at baseline, and prespecified major endpoints during the study period.

\section{Conclusions}

Our study underlines worse survival and cardiovascular outcomes in patients with type 2 diabetes and major PAD during 10 years of follow-up. Lower-extremity ulceration or amputation, and peripheral revascularization had similar effects on the risk of these outcomes. Furthermore, lower-extremity ulceration or amputation was associated with higher incidence of severe eye disease. Prompt screening and more intensive management of both presentations of major PAD may help to improve prognosis, particularly cardiovascular outcomes in patients with long-standing type 2 diabetes.

\section{Additional file}

Additional file 1: Table S1. Clinical characteristics of participants by history of major PAD at baseline. Table S2. Relative risk for outcomes during follow-up according to major PAD at baseline in participants free from history of macrovascular disease at baseline.

\section{Abbreviations}

ADVANCE: action in diabetes and vascular disease - preterax and diamicron modified-release controlled evaluation trial; AGE: advanced glycation endproducts; ANOVA: analysis of variance; BARI 2D: bypass angioplasty revascularization investigation 2 diabetes trial; $\mathrm{Cl}$ : confidence interval; CKD: chronic kidney disease; CKD-EPI: chronic kidney disease-epidemiology collaboration equation; eGFR: estimated glomerular filtration rate; ESRD: end stage renal disease; HR: hazard ratio; PAD: peripheral arterial disease; PROactive: PROspective pioglitAzone clinical trial in macrovascular events trial; RAGE: receptor of advanced glycation end-products; SD: standard deviation.

\section{Authors' contributions}

$\mathrm{KM}$ wrote the manuscript with assistance from $\mathrm{MW}$ and $\mathrm{JC} ; \mathrm{KM}, \mathrm{MW}$ and $\mathrm{JC}$ designed the study; $\mathrm{YH}$ contributed to statistical analyses and reviewed the manuscript; SZ, SC, PH, SH, NP, DRM and MM contributed to the discussion and reviewed the manuscript. JC and KM are the guarantors of this work and, as such, had full access to all the data in the study and take responsibility for the integrity of the data and the accuracy of the data analysis. All authors read and approved the final manuscript.

\footnotetext{
Author details

${ }^{1}$ The George Institute for Global Health, University of Sydney, PO Box M201, Missenden road, Camperdown, Sydney, NSW 2050, Australia. ${ }^{2}$ The George Institute for Global Health, University of Oxford, Oxford, UK. ${ }^{3}$ Department of Epidemiology, Johns Hopkins University, Baltimore, MD, USA. ${ }^{4}$ Monash Centre for Health Research and Implementation, School of Public Health and Preventive Medicine, Monash University, Clayton, VIC, Australia. ${ }^{5}$ Boden Institute of Obesity, Nutrition, Exercise and Eating Disorders, Sydney Medical School, University of Sydney, Sydney, NSW, Australia. ${ }^{6}$ Research Centre, Centre Hospitalier de I'Université de Montréal, Montreal, Canada. ${ }^{7}$ The University of Melbourne and Royal Melbourne Hospital, Melbourne, VIC, Australia. ${ }^{8}$ The International Centre for Circulatory Health, National Heart and Lung Institute, Imperial College, London, UK. ${ }^{9}$ Oxford Centre for Diabetes, Endocrinology and Metabolism, National Institute for Health Research, Oxford Biomedical Research Centre, Harris Manchester College, University of Oxford, Oxford, UK. ${ }^{10}$ INSERM, UMRS 1138, Centre de Recherche des Cordeliers, Paris, France. ${ }^{11}$ Department of Diabetology, Endocrinology and Nutrition,
}

Assistance Publique-Hôpitaux de Paris, Bichat Hospital, DHU FIRE, Paris, France. ${ }^{12}$ Université Paris Diderot, Sorbonne Paris Cité, UFR de Médecine, Paris, France.

\section{Acknowledgements}

Not applicable.

\section{Competing interests}

Dr. Kamel Mohammedi reports personal fees from Novo-Nordisk, outside the submitted work; Prof. Marc Woodward reports personal fees from Amgen, outside the submitted work; Prof. Pavel Hamet reports grants from Servier, during the conduct of the study; other from Servier, outside the submitted work; Prof. Stephen Harrap reports grants from National Health and Medical Research Council of Australia, grants from The George Institute for Global Health, during the conduct of the study; other from Servier, outside the submitted work; Prof. Neil Poulter reports grants from The George Institute, grants from British Heart Foundation/Diabetes UKTThe George Institute, during the conduct of the study; grants from The George Institute, grants from BHF/DUK/The George Institute, outside the submitted work; Prof. Michel Marre reports grants and personal fees from Novo Nordisk, grants and personal fees from Sanofi, grants and personal fees from Eli Lilly, personal fees from Servier, grants and personal fees from Merck Sharp and Dohme, personal fees from Abbott, grants and personal fees from Novartis, personal fees from Astra Zeneca, outside the submitted work; and Prof. John Chalmers reports grants from National Health and Medical Research Council of Australia, grants and personal fees from Servier, outside the submitted work. No other potential conflict of interest relevant to this article was reported.

\section{Availability of data and material}

The datasets analyzed during the current study are not publicly available due to consideration of intellectual property, due to many ongoing active collaborations worldwide, and to continuing analyses by the study investigators, but may be available from the principal investigator on reasonable request.

\section{Ethics approval and consent to participate}

The Institutional Ethics Committee of each participating centre approved the ADVANCE and ADVANCE-ON protocols, and all participants provided written informed consent.

\section{Funding}

Dr. Kamel Mohammedi was supported by grants from the Société Francophone du Diabète (SFD) and the Association Diabète Risque Vasculaire (ADRV).

Received: 9 July 2016 Accepted: 20 August 2016

Published online: 02 September 2016

\section{References}

1. Mahoney EM, Wang K, Keo HH, Duval S, Smolderen KG, Cohen DJ, Steg G, Bhatt DL, Hirsch AT. Reduction of atherothrombosis for continued health (REACH) registry investigators-vascular hospitalization rates and costs in patients with peripheral artery disease in the United States. Circ Cardiovasc Qual Outcomes. 2010;3(6):642-51.

2. Malyar N, Furstenberg T, Wellmann J, Meyborg M, Luders F, Gebauer $\mathrm{K}$, Bunzemeier $\mathrm{H}$, Roeder $\mathrm{N}$, Reinecke $\mathrm{H}$. Recent trends in morbidity and in-hospital outcomes of in-patients with peripheral arterial disease: a nationwide population-based analysis. Eur Heart J. 2013;34(341):2706-14.

3. Hirsch AT, Duval S. The global pandemic of peripheral artery disease. Lancet. 2013;382(9901I):1312-4.

4. Fowkes FG, Rudan D, Rudan I, Aboyans V, Denenberg JO, McDermott MM, Norman PE, Sampson UK, Williams LJ, Mensah GA, et al. Comparison of global estimates of prevalence and risk factors for peripheral artery disease in 2000 and 2010: a systematic review and analysis. Lancet. 2013;382(9901I):1329-40.

5. Marrett E, DiBonaventura M, Zhang Q. Burden of peripheral arterial disease in Europe and the United States: a patient survey. Health Qual Life Outcomes. 2013;11:175. 
6. Jones WS, Patel MR, Rockman CB, Guo Y, Adelman M, Riles T, Berger JS. Association of the ankle-brachial index with history of myocardial infarction and stroke. Am Heart J. 2014;167(41):499-505.

7. Emdin CA, Anderson SG, Callender T, Conrad N, Salimi-Khorshidi G, Mohseni H, Woodward M, Rahimi K. Usual blood pressure, peripheral arterial disease, and vascular risk: cohort study of 4.2 million adults. BMJ. 2015;351:4865.

8. Lin YS, Tung TH, Wang J, Chen YF, Chen TH, Lin MS, Chi CC, Chen MC. Peripheral arterial disease and atrial fibrillation and risk of stroke, heart failure hospitalization and cardiovascular death: a nationwide cohort study. Int J Cardiol. 2016:203:204-11.

9. Jude EB, Oyibo SO, Chalmers N, Boulton AJ. Peripheral arterial disease in diabetic and nondiabetic patients: a comparison of severity and outcome. Diabetes Care. 2001;24(8I):1433-7.

10. Selvin E, Erlinger TP. Prevalence of and risk factors for peripheral arterial disease in the United States: results from the National Health and Nutrition Examination Survey, 1999-2000. Circulation. 2004;110(6l):738-43.

11. Joosten MM, Pai JK, Bertoia ML, Rimm EB, Spiegelman D, Mittleman MA, Mukamal KJ. Associations between conventional cardiovascular risk factors and risk of peripheral artery disease in men. JAMA. 2012;308(16l):1660-7.

12. Shah AD, Langenberg C, Rapsomaniki E, Denaxas S, Pujades-Rodriguez M, Gale CP, Deanfield J, Smeeth L, Timmis A, Hemingway H. Type 2 diabetes and incidence of cardiovascular diseases: a cohort study in 1.9 million people. Lancet Diabetes Endocrinol. 2015;3(2):105-13.

13. Norman PE, Davis WA, Bruce DG, Davis TM. Peripheral arterial disease and risk of cardiac death in type 2 diabetes: the fremantle diabetes study. Diabetes Care. 2006;29(31):575-80.

14. Dormandy JA, Betteridge DJ, Schernthaner G, Pirags V, Norgren L. Impact of peripheral arterial disease in patients with diabetes-results from PROactive (PROactive 11). Atherosclerosis. 2009;202(11):272-81.

15. Abbott JD, Lombardero MS, Barsness GW, Pena-Sing I, Buitron LV, Singh P, Woodhead G, Tardif JC, Kelsey SF. Ankle-brachial index and cardiovascular outcomes in the bypass angioplasty revascularization investigation 2 diabetes trial. Am Heart J. 2012;164(4):585-90.

16. ADVANCE Management Committee. Study rationale and design of ADVANCE: action in diabetes and vascular disease-preterax and diamicron MR controlled evaluation. Diabetologia. 2001:44(9l):1118-20.

17. Zoungas S, Chalmers J, Neal B, Billot L, Li Q, Hirakawa Y, Arima H, Monaghan $\mathrm{H}$, Joshi R, Colagiuri S, et al. Follow-up of blood-pressure lowering and glucose control in type 2 diabetes. N Engl J Med. 2014;371(15I):1392-406

18. Patel A, MacMahon S, Chalmers J, Neal B, Woodward M, Billot L, Harrap S, Poulter N, Marre M, Cooper M, et al. Effects of a fixed combination of perindopril and indapamide on macrovascular and microvascular outcomes in patients with type 2 diabetes mellitus (the ADVANCE trial): a randomised controlled trial. Lancet. 2007;370(9590I):829-40.

19. Patel A, MacMahon S, Chalmers J, Neal B, Billot L, Woodward M, Marre M, Cooper M, Glasziou P, Grobbee D, et al. Intensive blood glucose control and vascular outcomes in patients with type 2 diabetes. N Engl J Med. 2008:358(241):2560-72.

20. Golledge J, Quigley F, Velu R, Walker PJ, Moxon JV. Association of impaired fasting glucose, diabetes and their management with the presentation and outcome of peripheral artery disease: a cohort study. Cardiovasc Diabetol. 2014:13:147.

21. Aboyans V, Desormais I, Lacroix P, Salazar J, Criqui MH, Laskar M. The general prognosis of patients with peripheral arterial disease differs according to the disease localization. J Am Coll Cardiol. 2010;55(91):898-903.
22. Chen Q, Smith CY, Bailey KR, Wennberg PW, Kullo IJ. Disease location is associated with survival in patients with peripheral arterial disease. J Am Heart Assoc. 2013;2(5I):e000304

23. Santoro L, Ferraro PM, Flex A, Nesci A, De Matteis G, Di Giorgio A, Zaccone V, Gambaro G, Gasbarrini A, Santoliquido A. New semiquantitative ultrasonographic score for peripheral arterial disease assessment and its association with cardiovascular risk factors. Hypertens Res. 2016. doi:10.1038/hr.2016.88.

24. Baba M, Davis WA, Norman PE, Davis TM. Temporal changes in the prevalence and associates of diabetes-related lower extremity amputations in patients with type 2 diabetes: the fremantle diabetes study. Cardiovasc Diabetol. 2015;14:152.

25. Rasmussen BS, Yderstraede KB, Carstensen B, Skov O, Beck-Nielsen H. Substantial reduction in the number of amputations among patients with diabetes: a cohort study over 16 years. Diabetologia. 2016:59(1I):121-9.

26. Brownrigg JR, Griffin M, Hughes CO, Jones KG, Patel N, Thompson MM, Hinchliffe RJ. Influence of foot ulceration on cause-specific mortality in patients with diabetes mellitus. J Vasc Surg. 2014;60(4):982-6.

27. Brownrigg JR, Davey J, Holt PJ, Davis WA, Thompson MM, Ray KK, Hinchliffe RJ. The association of ulceration of the foot with cardiovascular and all-cause mortality in patients with diabetes: a meta-analysis. Diabetologia. 2012;55(111):2906-12.

28. Schofield CJ, Libby G, Brennan GM, MacAlpine RR, Morris AD, Leese GP. Mortality and hospitalization in patients after amputation: a comparison between patients with and without diabetes. Diabetes Care. 2006;29(10l):2252-6.

29. Mohammedi K, Potier L, Belhatem N, Matallah N, Hadjadj S, Roussel R, Marre $M$, Velho $G$. Lower-extremity amputation as a marker for renal and cardiovascular events and mortality in patients with long standing type 1 diabetes. Cardiovasc Diabetol. 2016;15:5

30. Sigvant B, Kragsterman B, Falkenberg M, Hasvold P, Johansson S, Thuresson M, Nordanstig J. Contemporary cardiovascular risk and secondary preventive drug treatment patterns in peripheral artery disease patients undergoing revascularization. J Vasc Surg. 2016. doi:10.1016/j. jvs.2016.03.429.

31. Malmstedt J, Karvestedt L, Swedenborg J, Brismar K. The receptor for advanced glycation end products and risk of peripheral arterial disease, amputation or death in type 2 diabetes: a population-based cohort study. Cardiovasc Diabetol. 2015;14:93

32. Chen YW, Wang YY, Zhao D, Yu CG, Xin Z, Cao X, Shi J, Yang GR, Yuan MX, Yang JK. High prevalence of lower extremity peripheral artery disease in type 2 diabetes patients with proliferative diabetic retinopathy. PLoS ONE. 2015;10(3I):e0122022.

33. Mohammedi K, Woodward M, Hirakawa Y, Zoungas S, Williams B, Lisheng L, Rodgers A, Mancia G, Neal B, Harrap S, et al. Microvascular and macrovascular disease and risk for major peripheral arterial disease in patients with type 2 diabetes. Diabetes Care. 2016. doi:10.2337/dc16-0588.

34. Reddan DN, Marcus RJ, Owen WF Jr, Szczech LA, Landwehr DM. Longterm outcomes of revascularization for peripheral vascular disease in end-stage renal disease patients. Am J Kidney Dis. 2001;38(11):57-63.

35. Liew YP, Bartholomew JR, Demirjian S, Michaels J, Schreiber MJ Jr. Combined effect of chronic kidney disease and peripheral arterial disease on all-cause mortality in a high-risk population. Clin J Am Soc Nephrol. 2008;3(4I):1084-9.

36. Luders F, Bunzemeier H, Engelbertz C, Malyar NM, Meyborg M, Roeder N, Berger K, Reinecke H. CKD and Acute and Long-Term Outcome of Patients with Peripheral Artery Disease and Critical Limb Ischemia. Clin J Am Soc Nephrol. 2016;11(2l):216-22 